\title{
MISSING MEDICAL RECORDS: AN OBSTACLE TO ARCHIVAL SURVEY-RESEARCH IN A RURAL COMMUNITY IN SOUTH AFRICA
}

\begin{abstract}
Keeping good quality medical records is an essential yet often neglected part of a health-care practitioner's workload. In South Africa, by law all health care facilities are required to retain medical records for a minimum of six years after the cessation of a patient's treatment. In an archival survey that was attempted in a rural community in South Africa, only 39\% of the records that were requested were located. The procedure that was followed in order to obtain the records to be included in the survey is briefly described in this paper, highlighting the challenges experienced in four district hospitals in this community. The phenomenon has serious implications not only for the quality of healthcare, incidence of iatrogenic injuries and the future of the health-care practitioner's career, but it also impacts on the ability to conduct research to inform practice. An aspect that is not often considered is the impact of poor record keeping on the research and teaching component of the broader medical profession.
\end{abstract}

KEY WORDS: MEDICAL, RECORD KEEPING, ARCHIVAL RESEARCH.

\section{INTRODUCTION}

By law adequate medical records should be kept by all health professionals. These records contain valuable information regarding the patient's condition and management at the health-care institution(Health Professionals Council of South Africa (HPCSA, 2008). In addition medical records are a key source of quantitative information in social science research studies (HPCSA, 2008).

The purpose of keeping records is to ensure the continuity of care and to share relevant information with other members of the multi-disciplinary team (HPCSA, 2008; Claeys, 1996). Not only

\section{Correspondence Author:}

Liezel Wegner

Department of Physiotherapy

University of the Western Cape

Private Bag X 17

Bellville 7530

South Africa

Email: liwegner@uwc.ac.za is it imperative to keep records but the quality of the records should also be considered. A direct correlation has been found between the quality of records and quality of care at health-care facilities in the Netherlands (Zegers et al., 2009). This finding is supported by the South African Medical Protections Society (MPS) booklet which suggests that poor record keeping is a significant cause of adverse events and iatrogenic injuries during hospital stay (MPS, 2012).

Inadequate care could result in patient dissatisfaction with the healthservices delivered. The patient could thus potentially file a malpractice complaint against a health-care practitioner. In such cases, medical records also serve as a medico-legal document (HPCSA, 2008). According to the MPS casebook, inadequate record keeping can easily result in the end of a health-care practitioner's career if a complaint is made against the practitioner, and the medical records are insufficient to defend the practitioner's case (MPS, 2012).
The HPCSA has clear guidelines on how patient information should be recorded and retained, as well as the importance of maintaining and retaining these records. All health-care records should include the patient's general demographic details, records of the date and place of every consultation, relevant history, assessment, and the management of the patient (HPCSA, 2008; MPS, 2000). According to the HPCSA, one of the reasons for the retention of medical records is to promote teaching and research (HPCSA, 2008).

In South Africa, all private and public health-care facilities should retain patient records for at least six years (HPCSA, 2008). Records of minors should be kept until the age of 21 years and persons with mental impairments' records must be kept for the duration of their lifetime. All records of patients who were injured on duty should be kept for a period of 20 years after treatment ceased (HPCSA, 2008). The value of records for research purposes should also be evaluated before 
any records can be destroyed since medical records are frequently used as a source of quantitative information for research studies (Zegers et al., 2009; Raff \& James 2003). Records need to be legible, comprehensive and retained for researchers to be able to extract the necessary information from these records. In a South African survey on anaesthetic records done by Raff and James (2003), only $29.9 \%$ of the sample of 284 records was legible and complete. Missing data influences the validity and reliability of the findings and can result in missing data bias.

This article aims to demonstrate how missing records / data influenced archival research in four district hospitals in a rural community in South Africa. The intended archival survey formed part of a doctoral research project. The proposed archival survey was developed to provide baseline information regarding the incidence, demographic profile and causes of lower limb amputations of patients living in a rural community in South Africa. The methods used to obtain the patient folders necessary for the archival survey will be described and the difficulties and possible reasons for these difficulties will be presented under results and discussion.

\section{PROCEDURE OF ARCHIVAL DATA COLLECTION}

After ethical clearance to conduct the study was obtained from the ethics committee at the University of the Western Cape (UWC), four district hospitals that fall within a rural community in South Africa were conveniently selected. The records of all patients who received lower limb amputations between 1 January 2009 and 31 December 2010 at these hospitals were identified as the study population.

In order to identify the patients who suffered lower limb amputations, the theatre records at the selected hospitals were surveyed. Since a specific population's (amputees) records had to be retrieved, and no electronic database of patient's diagnosis were available, theatre records were scrutinized to identify all patients who received lower limb amputations in 2009 and 2010. Information recorded included the names of patients and wards that the patients were admitted, not the hospital folder numbers. Since the medical records are filed according to folder numbers, the folder number needed to be obtained in order to trace the folder in the records room. In order to determine patient folder numbers, the researcher had to use an alternative approach. Once the names of patients who had lower limb amputations in 2009 and 2010 were obtained from the theatre records, the patients' names and date of amputation were used to determine the hospital inpatient folder number from the wards' admission records. The only exception was for Hospital B. All amputations for patients serviced by this hospital were done at the regional hospital in the area since no orthopaedic surgeon was available at this specific district hospital to perform amputations during 2009 and 2010. Information about patients with lower limb amputations at this hospital was obtained from the Physiotherapy records since the theatre records would not reflect any amputations. The Physiotherapists at Hospital B assesses and manages all in-patients who received amputations. Once the folder number was obtained from the ward records, these names and folder numbers were then given to the respective hospitals' record clerks to obtain the folders from the records room. After the folders were obtained, the researcher scrutinized the records in order to complete the information on the data extraction sheet of the archival survey.

\section{ETHICAL CONSIDERATIONS}

Ethical considerations for research in social sciences were adhered to. Permission to conduct the research study was obtained from the University of the Western Cape's Senate Higher degrees Committee. Permission was obtained from the relevant provincial Department of Health, to conduct this study in government hospitals, as well as from the facility managers of the four district hospitals that were identified to be included in the study. A summary of the results of the research project will be disseminated to the various stakeholders involved.

\section{RESULTS}

\section{"Missing" records}

Between the four district hospitals according to the theatre records at Hospitals A, C and D, and the Physiotherapy records at Hospital B, 64 patients received lower limb amputations during the research period. However, many of these folders had to be excluded from the research (see Figure 1). A total of 22 (34\%) hospital folders could be located or were retained by the hospitals. Twenty folders contained information on the management of the patient. The two folders that were excluded contained no information on the management of the patient. Thus only 20 out of 64 (31\%) of all the records sought could be included in the research project.

\section{Hospital A}

For the period between 1 January 2009 and 31 December 2010, 25 persons received lower limb amputations at Hospital A according to the theatre records. Sixteen lower limb amputations were done in 2009 and nine in 2010. Of these 25 patients only 19 could be traced in the ward admissions records in order to establish the patients' hospital folder numbers. In total ten out of 19 records from the ward admission book were retrievable from the records room less than three years after end of treatment $(52.6 \%)$. Folders were filed in horizontal filing cabinets.

\section{Hospital B}

At this hospital 10 patients were admitted as in-patients after lower limb amputations. Four of these amputees were admitted in 2009 and six in 2010. Of the 10 patients identified as being treated in the wards of the hospital as in-patients (from the Physiotherapy records) only eight patients could be located in the ward admission records in order to trace their folder numbers. Only three out of the eight $(37.5 \%)$ patients' (for which folder numbers were available) records could be located. Out of the three folders that could be traced, two folders contained no information on the management of the patient, only demographic information. Out of 10 folders initially sought, only one 
contained medical information which could be used in the study. The record clerk did now allow the researcher to view the records room at Hospital B.

\section{Hospital C}

Twenty patients received lower limb amputations according to the theatre records, 11 in 2009 and nine in 2010. All twenty patients' folder numbers could be located but only five out of 20 $(25 \%)$ of these folders could be traced in the records room. Four folders dated from 2009 and only one from 2010. At this hospital all the records in the records room were lying in a heap on the floor of the records room and not filed in a methodical manner.

\section{Hospital D}

At Hospital D, nine patients received lower limb amputations. Six amputations were done in 2009 and three in 2010. Of these nine patients in-patient folder numbers could only be located for six of these patients from the ward admission records. Four out of nine (44\%) folders could be retrieved from the records room and were available to peruse. Three folders were retrieved with the right folder number but the names did not match the names of the patients who received amputations. Folders were filed in horizontal filing cabinets, but the cabinets were too full, and folders were bulging out of the cabinets and very difficult to retrieve.

\section{Incorrect documentation}

The first problem with incorrect documentation was the discrepancy between the theatre records and ward admission records. Routinely all patients that suffered an amputation will be admitted as an inpatient into a ward in the hospital. Of the 64 patients that had amputations according to the theatre records, only $53(83 \%)$ patients were admitted to the wards according to the ward admission records.

Incorrect documentation also proved to be an obstacle to locating folders. Five out of the $64(8 \%)$ folders were located according to their folder numbers but then presented with a different patient name than that of the patient requested.

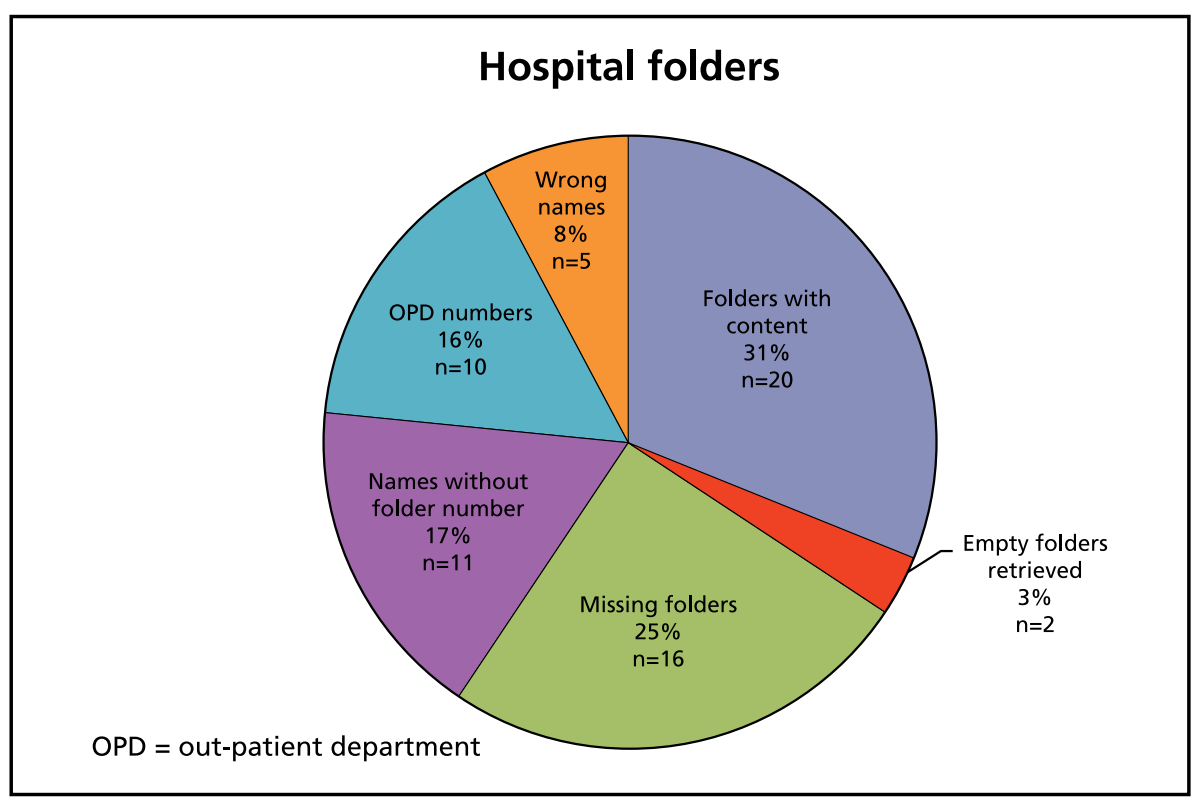

Figure 1: Possible reasons for folders being excluded from the archival survey $(n=64)$.

Table 1: Summary of folders obtained at each hospital.

\begin{tabular}{|l|l|l|l|l|l|}
\hline Hospital & $\begin{array}{l}\text { Number of } \\
\text { patients } \\
\text { identified to } \\
\text { be included }\end{array}$ & $\begin{array}{l}\text { Number } \\
\text { of patient } \\
\text { names } \\
\text { with folder } \\
\text { numbers }\end{array}$ & $\begin{array}{l}\text { Incomplete } \\
\text { folders }\end{array}$ & $\begin{array}{l}\text { Number } \\
\text { of folders } \\
\text { retrieved }\end{array}$ & $\begin{array}{l}\text { Number } \\
\text { of folders } \\
\text { used }\end{array}$ \\
\hline Hospital A & 25 & 19 & 0 & 10 & 10 \\
\hline Hospital B & 10 & 8 & 2 & 3 & 1 \\
\hline Hospital C & 20 & 20 & 0 & 5 & 5 \\
\hline Hospital D & 9 & 6 & 0 & 4 & 4 \\
\hline
\end{tabular}

\section{Different folder numbers}

Ten out of the $64(16 \%)$ of the patients that had amputations had "out-patient" folder numbers which could not be traced to a hospital folder number in order to obtain the folder containing the in-hospital management.

\section{Incomplete folders}

Another hindrance in obtaining data from the records was incomplete records. Two of the records that were located only contained demographic information of the patient and no information regarding the patient management, and one folder contained some information on patient management, but did not state that the patient had an amputation at all, even though it was recorded in the theatre records and the ward admissions book.

\section{Method of filing}

Lastly, three of the four hospitals subjectively seemed to have a lack of storage space for medical records. At one of the hospitals records were lying in piles on the floor and were not filed at all, and at the other two hospitals filing shelves were overloaded with records and records were wedged in amongst each other so tightly that it was very difficult to remove or replace a folder once it was removed from the shelve.

\section{DISCUSSION}

The aim of this paper was to demonstrate how inadequate record keeping can become an obstacle to research, since the archival survey could not be conducted due to an inability to retrieve the folders for a specific population 
group. This finding is resonated by the quote by Orbeta (n.d) 'My research was heavily hindered by poor documentation so I plan to educate the medical community about this issue to keep other valuable research from going to waste'.

Only $39 \%$ of the folders that were sought for use in the archival survey could be retrieved. Unfortunately no similar study could be found which reports on the difficulty locating folders. In this study only $3 \%(n=2)$ of the folders that were retrieved were incomplete. This finding compares well with the prospective survey that was conducted by Raff and James (2003) where $25 \%$ of the records contained no information of anaesthetic procedures and $45 \%$ of the total sample was incomplete or illegible.

Little attention is being paid to the folder number when a new patient is admitted to the hospital. A patient might be seen initially as an out-patient, and have an out-patient folder number. If this patient is then admitted into the hospital a new folder might not be opened and the patient's will continue using his outpatient folder number. These numbers are very similar but the in-patient and out-patient folders are normally filed and stored separately. The ward admission records will then record the folder number but not realise or specify that it is an out-patient number. This folder will then be difficult to trace in the in-patient records room.

The incorrect or non-documentation in the hospital ward admission records could be considered a major barrier to locating patient folders. Eleven of the patients, who suffered lower limb amputations according to the theatre notes, were not recorded as being admitted as in-patients in the wards admission books. No electronic records of patients and folder numbers were kept at any of these district hospitals, which makes locating a specific population of patients' hospital folders a tedious task and greatly reliant on accurate manual record keeping. Electronic record keeping could possibly ease the task of obtaining a specific sample of records for research purposes (Orbeta, n.d).

The challenge this filing system could potentially present is that when a patient came for a follow up visit as an outpatient after he was hospitalised and did not have his hospital folder number, a new folder would be opened for the patient. The health-care practitioner attending to this patient as an outpatient would then have no information regarding the history and previous management of this patient. This finding directly opposed the principle motive for keeping medical records (HPCSA, 2008). As a result one patient might have numerous folders which impedes on the limited storage space for medical records at health-care facilities, and this could directly lead to adverse events or iatrogenic injuries (MPS, 2012).

Another challenge was that even though the folder number might have been obtained the researcher would be given a folder with a matching folder number but a different patient name and condition. This faulty record-keeping could easily cause mismanagement if the practitioner did not check the patient's name, resulting in adverse events or iatrogenic injury (Zegers et al., 2009; MPS, 2000).

Records clerks were always very busy servicing patients awaiting their folders in order to be seen by a health-care practitioner. The low number of records that could be located could subjectively possibly be a result of the clerks already being overworked and not having an efficient filing system which would ease the manual location of records (Clayes, 1996).

Only $39 \%$ of the records that were sought for the intended archival survey in 2011 were available and accessible less than two years after treatment (2009 and 2010). No statistically significant conclusions could be drawn due to the small sample size, thus affecting the quality of the research. These four hospitals did not comply with the HPCSA guidelines stating that all medical records should be kept for at least six years after treatment (HPCSA, 2008). If the records are not adequately retained it cannot be reviewed in case of a query, and it cannot be utilised for research purposes. The records obtained in this specific sample also did not comply with the HPCSA's specifications on the minimum information that should be included in the medical records. Three of the 22 folders that were retrieved had no information other than the patient's demographic details in it.

Medical research is vital for teaching purposes as well as determining common trends in disease and disability (Bonita, Beaglehole \& Kjellstrom, 2006). Having access to medical records to review is imperative as these are valuable sources of data. The information obtained from medical records enables the health-care providers to plan and implement preventative measures to improve the health of the overall population (Bonita, Beaglehole \& Kjellstrom, 2006). Secondly research is essential to identify problems in health-care service delivery to facilitate improvement of health-care systems (HPCSA, 2008).

\section{CONCLUSION}

In conclusion, inadequate record keeping was found to be a major obstacle to doing archival research in a rural community in South Africa. An inability to retrieve the necessary medical records hindered the research to such an extent that the study had to be delayed by nearly a year and changed to a prospective survey. This change has implications for human resources at the hospitals, and has financial implications which can further hamper the research.

Record keeping is the lawful responsibility of every health-care practitioner, and retention of medical records the duty of all health-care facilities. Inadequate record keeping can compromise the health of the patient as well as the career of the health-care practitioner. It is also a major obstacle in archival survey research as were the case demonstrated in this paper. Lack of or poor medical research will in turn affect the quality of medical teaching and the quality of health-care services negatively. According to Pourasghar et al 2008, it is necessary to find ways to ensure that the documentation of information will be in a readable and retrievable format. 


\section{REFERENCES}

Bonita R, Beaglehole R, Kjellstrom T. (2006). WHO Basic epidemiology, $2^{\text {nd }}$ Edition. Geneva Switzerland: WHO Press.

Claeys T. (1996). Medical filing, $2^{\text {nd }}$ Edition. Delmar Cengage Learning.

HPCSA (2008). HPCSA guidelines for good practice in the health care professions: Guidelines on the keeping of patient records. Booklet 14. Pretoria. Retrieved 15 May 2012 from: http://www.hpcsa.co.za/downloads/conduct_ ethics/rules/generic_ethical_rules/booklet_14 keeping_of_patience_records.pdf

Medical protection society (2000). Making and keeping medical records. MPS Casebook 13 (International). Retrieved 11 May 2012 from: http://www.medicalprotection.org/Default.aspx? $\mathrm{DN}=1843 \mathrm{c} 3 \mathrm{ce}-9570-41 \mathrm{f3}-\mathrm{b} 9 \mathrm{f1}-11 \mathrm{bfdc} 2138 \mathrm{ba}$
Medical protection society (2012). MPS Casebook 20 (International). Retrieved on 11 May 2012 from: http://www.medicalprotection org/Default.aspx? $\mathrm{DN}=\mathrm{f0c10d67-a680-44a7-a599-ba1b3c7384 \textrm {e } 0}$

Orbeta P. (n.d). Poor medical documentation: Its Consequences and future considerations. Final report for Carnegie Mellon University. Retrieved 12 May from: http://www.contrib.andrew.cmu. edu/ porbeta/files/67390 final report.pdf

Pourasghar F, Malekafzali H, Kazemi A, Ellenius J \& Fors U. (2008). What they fill in today, may not be useful tomorrow: Lessons learned from studying Medical Records at the Women hospital in Tabriz, Iran. BMC Public Health, 8:139.

Raff M and James MFM (2003). An audit of anaesthetic record keeping. Southern African journal of anaesthesia \& analgesia - July (p. 7-9).
Zegers M, de Bruijne MC, Spreeuwenberg P, Wagner C, Groenewegen PP, van der Wal G. (2009). Quality of patient record keeping: an indicator of the quality of care? BMJ Qual Saf. Retrieved 12 May 2012 from: http://qualitysafety.bmj.com/ content/early/2011/02/08/bmjqs.2009.038976. abstract 4343 